\title{
Fast MR arthrography using VIBE sequences to evaluate the rotator cuff
}

\author{
Jan E. Vandevenne • Filip Vanhoenacker • \\ Jestinah M. Mahachie John • Geert Gelin • \\ Paul M. Parizel
}

Received: 3 December 2008 /Revised: 17 February 2009 / Accepted: 27 February 2009/Published online: 18 March 2009

(C) ISS 2009

\begin{abstract}
Purpose The purpose of this paper was to evaluate if short volumetric interpolated breath-hold examination (VIBE) sequences can be used as a substitute for T1-weighted with fat saturation (T1-FS) sequences when performing magnetic resonance (MR) arthrography to diagnose rotator cuff tears. Materials and methods Eighty-two patients underwent direct MR arthrography of the shoulder joint using VIBE (acquisition time of $13 \mathrm{~s}$ ) and T1-FS (acquisition time of $5 \mathrm{~min}$ ) sequences in the axial and paracoronal plane on a 1.0-T MR unit. Two radiologists scored rotator cuff tendons on VIBE and T1-FS images separately as normal, small/ large partial thickness and full thickness tears with or without geyser sign. T1-FS sequences were considered the gold standard. Surgical correlation was available in a small sample.

Results Sensitivity, specificity, and positive and negative predictive values of VIBE were greater than $92 \%$ for large articular-sided partial thickness and full thickness tears. For detecting fraying and articular-sided small partial thickness tears, these parameters were $55 \%, 94 \%, 94 \%$, and $57 \%$,
\end{abstract}

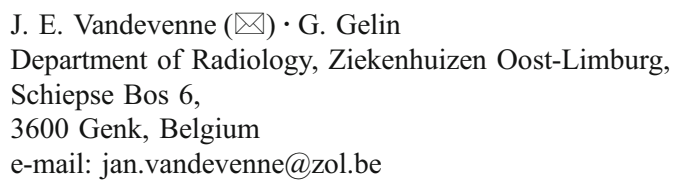

J. E. Vandevenne $\cdot$ F. Vanhoenacker $\cdot$ P. M. Parizel Department of Radiology, Universitair Ziekenhuis Antwerpen, University of Antwerp,

Wilrijkstraat 10,

2650 Edegem, Belgium

J. M. Mahachie John

Centre for Statistics, University of Hasselt,

Campus Diepenbeek, Agoralaan, Building D,

3590 Diepenbeek, Belgium respectively. The simple kappa value was 0.76 , and the weighted kappa value was 0.86 for agreement between T1FS and VIBE scores. All large partial and full thickness tears at surgery were correctly diagnosed using VIBE or T1-FS MR images.

Conclusion Fast MR arthrography of the shoulder joint using VIBE sequences showed good concordance with the classically used T1-FS sequences for the appearance of the rotator cuff, in particular for large articular-sided partial thickness tears and for full thickness tears. Due to its very short acquisition time, VIBE may be especially useful when performing MR arthrography in claustrophobic patients or patients with a painful shoulder.

Keywords MR arthrography - Shoulder joint - Rotator cuff . Gradient echo sequence

\section{Introduction}

Magnetic resonance (MR) arthrography of the shoulder joint has proven to be an excellent diagnostic tool for evaluating internal derangements including rotator cuff tears. The standardly used MR technique after gadolinium contrast injection into the shoulder joint includes spin echo T1-weighted sequences with fat saturation (T1-FS) applied in different planes, and the total examination lasts at least 10-15 min to perform [1-7].

Patients suffering from recent trauma involving the shoulder joint often are in pain when presenting for the MR arthrography examination and have difficulty to lie still for more than a few minutes. The latter also holds true for many claustrophobic patients. Applying T1-FS sequences in these patients may result in images that cannot be interpreted due to motion artifacts. 
Gradient echo sequences such as volumetric interpolated breath-hold examination (VIBE) were shown to provide high signal-to-noise ratios for a range of gadolinium concentrations comparable to gadolinium concentrations optimal for T1-FS sequences [8]. The VIBE sequence can be adjusted to last only little more than $10 \mathrm{~s}$.

Before starting to use VIBE sequences instead of T1-FS sequences for MR arthrography of the shoulder to image patients that are unable to lie still for a prolonged time within the MR unit, the accuracy of VIBE sequences should be tested in a group of patients that are lying perfectly still rendering adequate T1-FS images for comparison. Therefore, the purpose of this study was to evaluate if short VIBE sequences can be used as a substitute for T1-FS sequences when performing MR arthrography to diagnose rotator cuff tears.

\section{Materials and methods}

Eighty-two patients were consecutively, over a period of 6 months, included in this prospective study after referral by their orthopedic surgeon for MR arthrography of the shoulder joint (mean age of 44 years, ranging between 15 and 77 years, and consisting of 39 women and 43 men). Approval for this study was waived by the ethics committee of our hospital.

In each patient, one glenohumeral joint was injected using a 21 gauge needle with a mixture of gadolinium chelated (0.1 ml Dotarem, Guerbet, Roissy, France) and iodinated contrast medium (10 ml Hexabrix, Guerbet, Paris, France), followed by $1-5 \mathrm{ml}$ saline to achieve sufficient capsular distension. After this fluoroscopically guided procedure using the anterior approach, patients were transferred to the MR unit (Harmony, 1.0 Tesla, Siemens, Erlangen, Germany) for imaging using a dedicated shoulder transmit-receiver coil. Both a VIBE sequence and a T1-FS sequence were applied in the transverse and paracoronal planes. The parameters of the T1-FS sequence were as follows: TR/TE 880/12 ms, spectral fat saturation, field-of-view (FOV) of $230 \times 211$, matrix of $384 \times 264$, slice thickness of $3 \mathrm{~mm}$ with a $10 \%$ interslice gap, and one acquisition. This resulted in a voxel volume of $0.8 \times$ $0.6 \times 3 \mathrm{~mm}\left(1.44 \mathrm{~mm}^{3}\right)$. The parameters of the VIBE sequence were: TR/TE $4.45 / 1.86 \mathrm{~m}$, flip angle $12^{\circ}$, FOV of $200 \times 163 \mathrm{~mm}$, matrix of $120 \times 256$, one slab of 40 slices with slice thickness of $2 \mathrm{~mm}$, and one acquisition. This resulted in a voxel volume of $1.4 \times 0.8 \times 2 \mathrm{~mm}\left(2.24 \mathrm{~mm}^{3}\right)$. Total acquisition time of one T1-FS sequence was $5 \mathrm{~min}$ and of one VIBE sequence was 13 s. Patients whose images showed motion artifacts on the T1-FS sequences were not included.

The MR arthrography exams were scored in a blinded fashion by two reviewers (experienced musculoskeletal radiologists) to evaluate for rotator cuff tears. Each reviewer separately scored all MR arthrography exams using only one set of images (T1-FS or VIBE images) and 2 weeks later only the other set. Differences in scoring between the two reviewers were solved by consensus. A normal rotator cuff was scored as 1, fraying and small partial thickness tears on the articular side of the rotator cuff as 2, large or subtotal partial thickness tears on the articular side of the rotator cuff as 3, full thickness tears of the rotator cuff as 4, and full thickness tears with contrast extension into the acromioclavicular joint (geyser sign) as 5. Differentiation between small and large partial thickness tears was based on tear depth, corresponding to a modification of the Ellman classification [9, 10]: a small partial thickness tear was defined as involving one third or less of the thickness of the rotator cuff at that particular location, and a large partial thickness tear as more than one third of the thickness of the rotator cuff. The location of the rotator cuff tears was noted to be in the subscapular, supraspinatus, infraspinatus, or teres minor tendons. The highest score given to one of these four parts of the rotator cuff was used as the score for the rotator cuff as a whole. SAS software (version 6.12, SAS Institute, Cary, NC, USA) was used for statistical analysis. Sensitivity, specificity, positive predictive value, and negative predictive value for scoring rotator cuff tears using the VIBE sequence were calculated considering the T1-FS sequence as the gold standard. The kappa coefficient was calculated to describe the strength of agreement between the scores of VIBE and T1-FS. The higher the kappa value, the stronger the agreement, e.g., values between $0.61-0.80$ indicate good agreement, and values higher than 0.81 indicate very good agreement [11]. The weighted kappa coefficient was also calculated because the scores were ordered from smaller to larger tears, and this weighted kappa value takes in account that adjacent scores are closer to agreement than scores far apart [12]. Furthermore, the kappa coefficient as a measure for interobserver agreement between the two readers was calculated, both for the scoring of the T1-FS images and the VIBE images.

Clinical records were reviewed, and if available, surgical reports were evaluated for description of the rotator cuff. The surgical data were correlated with the findings at VIBE and T1-FS MR images, considering the scores for the rotator cuff as a whole.

\section{Results}

The results were summarized in Tables 1, 2, and 3. In these 82 patients, a total of 97 tendon tears were diagnosed on T1-FS images, whereas 79 tears were seen on VIBE images. Mean size of full thickness tears was $17 \times 19 \mathrm{~mm}$ 
Table 1 Distribution of scored rotator cuff tears on T1-FS and VIBE images in 82 patients

Numbers refer to the number of rotator cuff tendons

\begin{tabular}{|c|c|c|c|c|c|c|}
\hline \multirow[t]{2}{*}{ Score } & \multicolumn{2}{|c|}{ Supraspinatus } & \multicolumn{2}{|c|}{ Infraspinatus } & \multicolumn{2}{|c|}{ Subscapularis } \\
\hline & T1-FS & VIBE & T1-FS & VIBE & T1-FS & VIBE \\
\hline 1 (normal) & 24 & 37 & 64 & 67 & 61 & 63 \\
\hline 2 (small partial thickness) & 29 & 17 & 4 & 1 & 6 & 6 \\
\hline 3 (large partial thickness) & 14 & 14 & 2 & 2 & 11 & 9 \\
\hline 4 (full thickness) & 10 & 9 & 8 & 8 & 4 & 4 \\
\hline 5 (geyser sign) & 5 & 5 & 4 & 4 & 0 & 0 \\
\hline
\end{tabular}

(ranging between $4 \times 4$ and $42 \times 40 \mathrm{~mm}$ ) in transverse and longitudinal direction, respectively. Distribution of tears per tendon was presented in Table 1. Scores from the teres minor tendon were not displayed since the two sequences had a total agreement that all patients had a normal teres minor tendon. False negative readings on VIBE mainly occurred for fraying and small partial thickness tears (score 2) in the suprapinatus and infraspinatus tendons.

Considering the rotator cuff as a whole, 65 torn rotator cuffs were diagnosed on T1-FS, whereas 54 torn cuffs were seen on VIBE. Cross tabulation of the scores of the rotator cuff as a whole demonstrated that the highest number of rotator cuffs are along the diagonal, which may imply a "good" agreement between T1-FS and VIBE scores, in particular for scores 3 (large partial thickness tears) and higher (Table 2).

This was confirmed in the results of statistical analysis (Table 3). Sensitivity, specificity, and predictive values of VIBE were greater than $92 \%$ for large partial thickness tears (VIBE/T1-FS, 19/20) and full thickness tears with $(5 / 5)$ or without geyser sign (12/13). Regarding fraying and small partial thickness tears $(15 / 27)$, sensitivity and negative predictive value were relatively low ( $56 \%$ and $57 \%$ ); however, specificity and positive predictive value remained high (above 93\%).

The simple kappa value was 0.76 (95\% confidence interval 0.66-0.87) indicating good agreement of VIBE scores and T1-FS scores of the rotator cuff. This index further increased to very good agreement when taking in account the order of the scores: weighted kappa value of 0.86 (95\% confidence interval 0.80-0.93). Between the two readers, ten MR examinations (five T1-FS and five VIBE MR examinations) required consensus review: nine of ten consensus reviews were necessary for discrepancies between scores 1 and 2 (normal versus fraying or small partial thickness tear). For the T1-FS MR examinations, the kappa value for interobserver agreement was 0.90 (95\% confidence interval 0.83-0.98); for the VIBE examinations, the kappa value for interobserver agreement was 0.94 (95\% confidence interval $0.87-0.99$ ).

Clinical records were available in 51 patients. Twentynine patients did not have surgery. From the 22 patients undergoing surgery, four patients had acromioplasty only, and the rotator cuff was not thoroughly inspected. In the remaining 18 patients, the surgeons diagnosed eight full thickness tears, three large partial thickness tears, and one cuff with fraying at the undersurface. Six rotator cuffs were intact at surgery. All tears were correctly diagnosed both on T1-FS and on VIBE MR images. On T1-FS MR images, two patients were diagnosed false positively with fraying of the rotator cuff.

\section{Discussion}

Direct MR arthrography using T1-FS sequences is known as an excellent imaging technique to diagnose internal derangements of the shoulder joint but suffers from prolonged acquisition times of several minutes. Typically, a treating physician refers the patient for an MR arthrography to the radiology department, contrast medium is injected into the glenohumeral joint, and subsequently, the patient is presented to the MR unit. However, some patients in pain after recent shoulder joint injury or claustrophobic patients may not be able to lie still for several minutes. In our experience, the T1-FS images rendered from these patients often are not interpretable because motion artifacts degrade the image quality. Therefore, MR sequences lasting in the range of seconds instead of minutes could be helpful to return diagnostic information in these patients, at least regarding the status of the rotator cuff.

Table 2 Cross tabulation of highest score for each rotator cuff on T1FS and VIBE images

\begin{tabular}{lrrrrrr}
\hline T1-FS score & \multicolumn{6}{l}{ VIBE score } \\
\cline { 2 - 6 } & 1 & 2 & 3 & 4 & 5 & Total \\
\cline { 2 - 6 } 1 & 16 & 1 & 0 & 0 & 0 & 17 \\
2 & 12 & 15 & 0 & 0 & 0 & 27 \\
3 & 0 & 1 & 19 & 0 & 0 & 20 \\
4 & 0 & 0 & 1 & 12 & 0 & 13 \\
5 & 0 & 0 & 0 & 0 & 5 & 5 \\
Total & 28 & 17 & 20 & 12 & 5 & 82 \\
\hline
\end{tabular}

Numbers refer to the number of rotator cuffs 
Table 3 Efficacy of VIBE in the diagnosis of rotator cuff tears compared to T1-FS

$C I$ confidence interval

\begin{tabular}{lllll}
\hline VIBE & score 2 & score 3 & score 4 & score5 \\
\hline Sensitivity (\%) & 55.56 & 95 & 92.31 & 100 \\
$95 \%$ CI & $0.36-0.74$ & $0.73-1$ & $0.62-1$ & $0.46-1$ \\
Specificity (\%) & 94.12 & 100 & 100 & 100 \\
$95 \%$ CI & $0.69-1$ & $0.76-1$ & $0.76-1$ & $0.76-1$ \\
Positive predictive value (\%) & 93.75 & 100 & 100 & \\
$95 \%$ CI & $0.68-1$ & $0.79-1$ & $0.70-1$ & \\
Negative predictive value (\%) & 57.14 & 94.12 & 94.12 & \\
$95 \%$ CI & $0.37-0.75$ & $0.76-1$ & $0.69-1$ & \\
\hline
\end{tabular}

VIBE is a gradient echo sequence with T1-weighting (fast low angle shot or FLASH technique), a high sensitivity for gadolinium-enhanced areas, and acquisition times that can be as short as a few seconds. Therefore, VIBE is mainly used for abdominal breath-hold imaging applications, e.g., to study the enhancement of abdominal organs after intravenous gadolinium injection [13]. VIBE has been used to image other body parts, but to our knowledge, no reports evaluated its accuracy to diagnose rotator cuff tears [14-17]. According to a experimental study, the signal-to-noise ratio profile versus gadolinium concentration of VIBE sequences is in the same range as for the commonly used T1-FS sequences on 1.5 and 3.0 T, enabling the use of VIBE sequences at the same time in the same patient as T1-FS sequences [8].

In our study, the VIBE sequence was tuned to last only $13 \mathrm{~s}$ while maintaining acceptable image quality. Considering T1-FS as the gold standard imaging sequence, the results showed that the VIBE sequence was an excellent tool for diagnosing large partial thickness and full thickness tears with or without geyser sign (Figs. 1, 2, and 3). The decreased accuracy of VIBE for diagnosing fraying and small partial thickness tears was felt to be related to the lower spatial resolution. This might be sustained by the fact that partial thickness tears were less sharply delineated on VIBE compared to T1-FS. On VIBE, partial thickness tears were often only recognized by a focal bulge with obtuse margins of the contrast medium into the rotator cuff (Fig. 2).

Considering surgery as the gold standard, T1-FS and VIBE sequences accurately diagnosed all rotator cuff tears in the subset of patients with clinical/surgical records available. False positive results for fraying of the rotator cuff on T1-FS MR images may indicate that the interpreters scored any small irregularity of the rotator cuff delineation as fraying on the imaging gold standard. The latter may constitute another explanation for the difference in concordance between VIBE and T1-FS MR images regarding fraying of the rotator cuff.

Rotator cuff tears can be accurately diagnosed using ultrasound [18]. Being of quick and inexpensive nature, ultrasound is frequently used as first imaging modality to evaluate the rotator cuff, also in patients suffering from painful shoulders or from claustrophobia. Conventional MR imaging may be used to image the rotator cuff, but it has been demonstrated that MR arthrography is more accurate for diagnosing rotator cuff tears [7]. When chosen to have MR arthrography, some patients appear not able to lie still
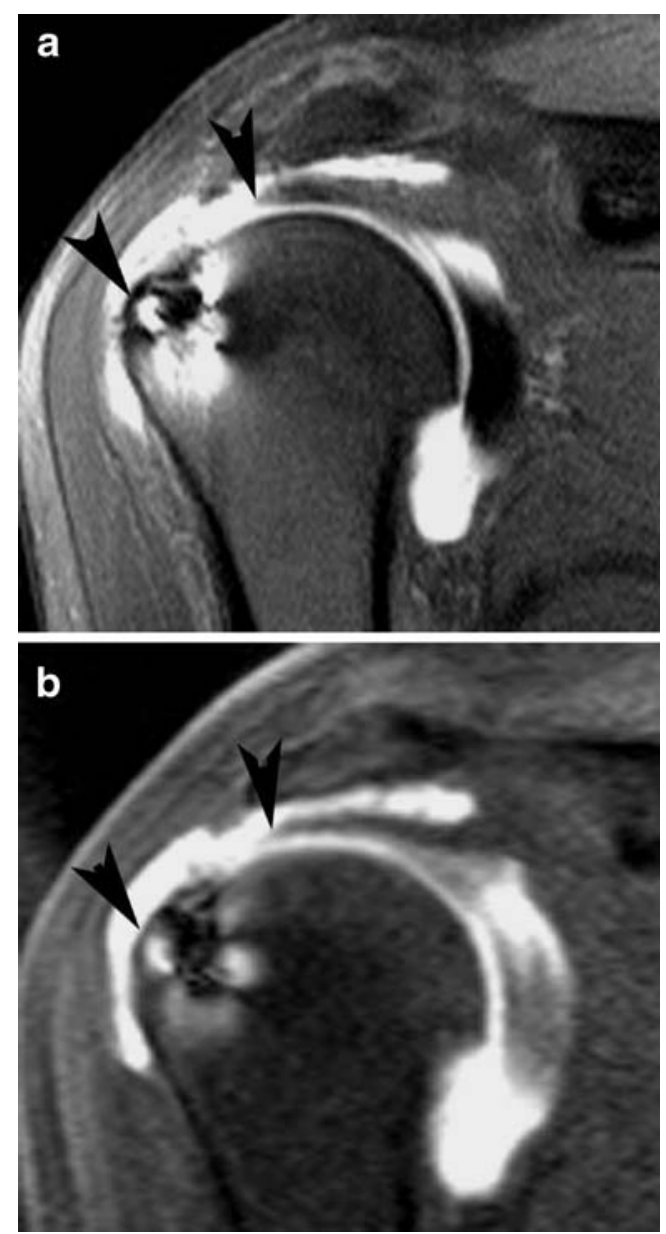

Fig. 1 Full thickness tear (arrowheads) of the infraspinatus tendon seen by contrast leakage from the glenohumeral joint into the subacromiosubdeltoid bursa, both on T1-FS (a) and VIBE (b) images. Metal artifacts from previous surgery 

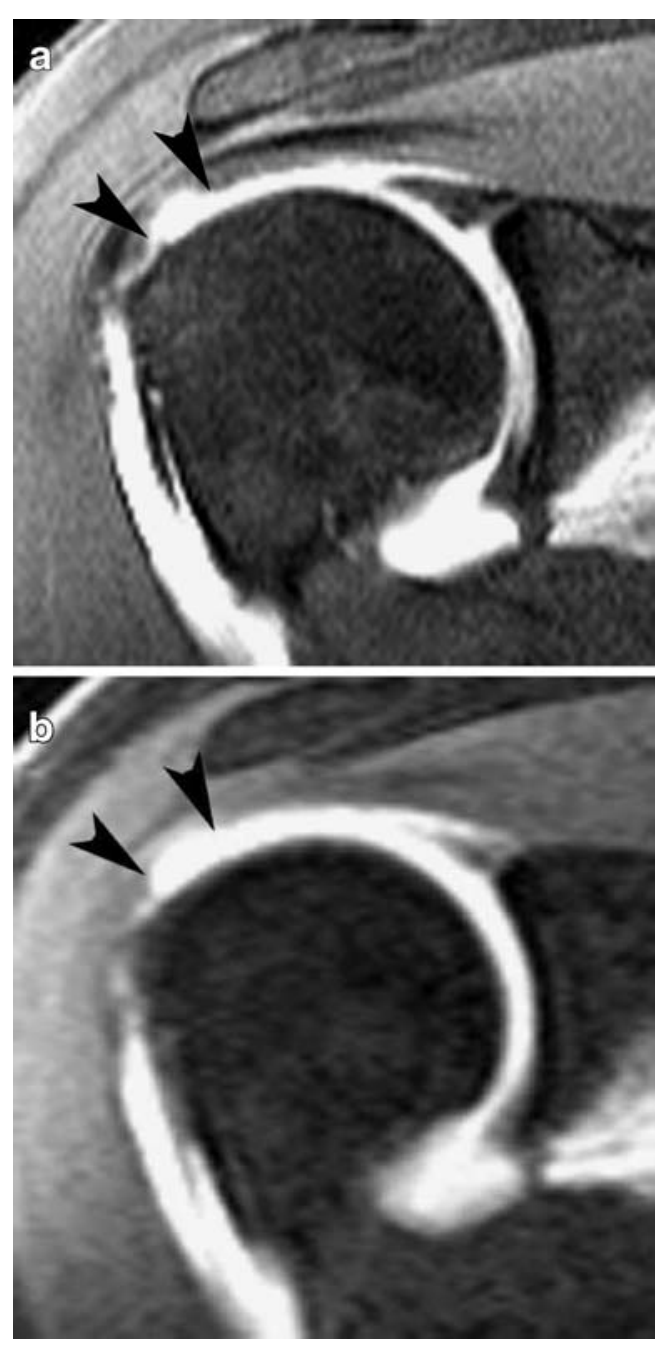

Fig. 2 Large partial thickness undersurface tear of the supraspinatus tendon (arrowheads) scored on the T1-FS (a) and the VIBE images (b). Note that the tear appeared rather as an added bulge of contrast medium into the supraspinatus tendon on VIBE, whereas the tear is sharper delineated on T1-FS

in the MR unit, only after their shoulder joint already has been injected. Up till now in our department, these patients could immediately be switched to computerized tomography (CT) arthrography because a large amount of iodine was present in the contrast medium mixture we used. CT arthrography using multidetector arrays can provide precise answers to all pre-operative questions (location and extent of the tear, position of the retracted tendon, and muscle atrophy), but MR arthrography seems to be the method of choice for the pre-operative workup of suspected rotator cuff pathology [19]. On the other hand, the use of large quantities of iodine for MR arthrography is not advocated because the presence of iodine in gadolinium-enhanced synovial fluid may produce a pronounced signal reduction on MR imaging $[8,20]$. Currently in our department, we have decreased the amount of iodine injection to a few drops to verify needle tip position using fluoroscopy [21];
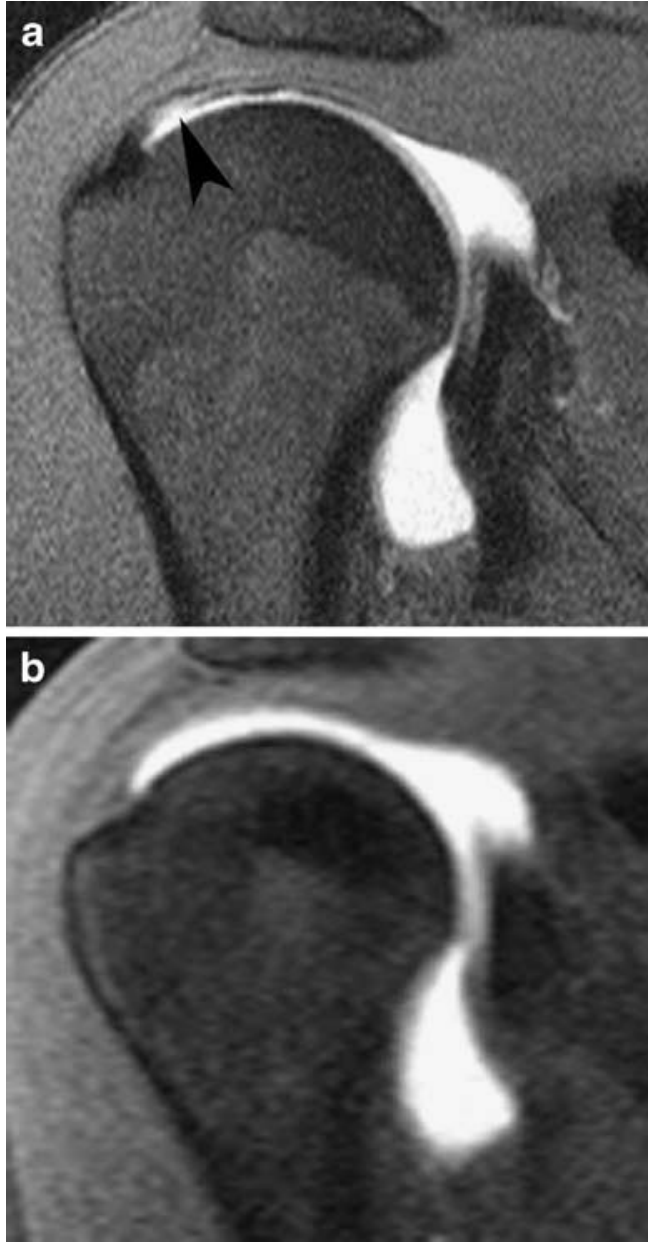

Fig. 3 Fraying and small partial thickness undersurface tear (arrowhead) of the supraspinatus tendon readily seen on the T1-FS image (a) but not appreciated on the VIBE image (b)

however, it cancels the option to switch to CT arthrography in patients that are unable to lie still in the MR unit. In these patients, we replace the standard T1-FS sequences by fast VIBE sequences to obtain information on the status of the rotator cuff.

From a surgical perspective, imaging of the rotator cuff may influence the decision to proceed to surgery. In particular, acute full thickness tears may require surgery. Acute partial thickness tears may be managed nonsurgically or surgically depending on associated clinical findings. Degenerative rotator cuff failure and fraying are usually managed nonsurgically [22]. Although VIBE sequences demonstrated low accuracy to diagnose fraying of the rotator cuff, large partial and full thickness tears were diagnosed correctly. Therefore, we feel comfortable to use VIBE sequences to assist the surgeon in selecting rotator cuff lesions that may require surgery or not, in particular for patients that cannot lie still for a prolonged imaging time.

Several limitations to this study should be mentioned. We used a 1.0-T MR unit, although 1.5 T MR units are 
currently widely available and routinely used for MR arthrography. However, a study on the accuracy of MR arthrography to evaluate the rotator cuff using a 1.0-T MR unit was recently published [9]. Moreover, claustrophobic patients (VIBE may especially be useful for these patients) may benefit from being imaged in open MR units, which currently have magnet fields up to $1.0 \mathrm{~T}$ instead of $1.5 \mathrm{~T}$. In this study, only axial and coronal planes were used to evaluate the rotator cuff. Sagittal planes and images acquired in the abduction-external rotation position were not studied, although these additional sequences are known to be of value for detecting (partial thickness) rotator cuff tears [23]. Bursal-sided partial thickness tears nor fatty infiltration and atrophy of the rotator cuff muscle bellies could be diagnosed using these VIBE sequences. Other intra-articular structures such as the labrum, biceps tendon, and glenohumeral ligaments and cartilage were not evaluated in this study, although lesions of these structures are also important and may co-exist in the same patient. In the future, adjusting the VIBE sequence to visualize these smaller intra-articular structures may be achieved by increasing spatial resolution and signal-to-noise ratio, i.e., increasing the image matrix and the number of acquisitions or using MR units with higher magnetic field strength. Recently, gradient echo sequences with longer acquisition times for shoulder MR arthrography were reported to have good accuracy to diagnose labral tears [24, 25]. Eventually, $3 \mathrm{D}$-gradient echo sequences may produce images useful for diagnostic virtual arthroscopy of the shoulder joint [26].

In conclusion, this study on MR arthrography of the shoulder joint showed good concordance between the appearance of the rotator cuff on VIBE and T1-FS sequences in particular for large partial and full thickness tears. Due to its very short acquisition time, VIBE may be useful as a substitute for the standard used T1-FS sequences when performing MR arthrography to evaluate the rotator cuff, especially in claustrophobic patients or in patients suffering from pain after recent injury.

\section{References}

1. Chung CB, Corrente L, Resnick D. MR arthrography of the shoulder. Magn Reson Imaging Clin N Am 2004;2: 25-38.

2. Meister K, Thesing J, Montgomery WJ, Indelicato PA, Walczak S, Fontenot W. MR arthrography of partial thickness tears of the undersurface of the rotator cuff: an arthroscopic correlation. Skeletal Radiol 2004;33: 136-141.

3. Vanhoenacker P, Vanhoenacker F, Crevits I, Simons P, Van Dyck P. MR arthrography of the rotator cuff and capsulolabral complex. JBR-BTR 2000;83: 313-318.

4. Sahin G, Demirtas M. An overview of MR arthrography with emphasis on the current technique and applicational hints and tips. Eur J Radiol 2006;6: 416-430.
5. Palmer WE, Brown JH, Rosenthal DI. Rotator cuff: evaluation with fat-suppressed MR arthrography. Radiology 1993;9: 683-687.

6. Morag Y, Jacobson JA, Miller B, De Maeseneer M, Girish G, Jamadar D. MR imaging of rotator cuff injury: what the clinician needs to know. Radiographics 2006;26: 1045-1065.

7. Magee T, Williams D, Mani N. Shoulder MR arthrography: which patient group benefits most? Am J Roentgenol 2004;183: 969-974.

8. Andreisek G, Froehlich JM, Hodler J, et al. Direct MR arthrography at $1.5 \mathrm{~T}$ and 3.0 T: Signal dependence on gadolinium and iodine concentrations-Phantom Study. Radiology 2008;247: 706-716.

9. Waldt S, Bruegel M, Mueller D, et al. Rotator cuff tears: assessment with MR arthrography in 275 patients with arthroscopic correlation. Eur Radiol 2007;17: 491-498.

10. Ellman H. Diagnosis and treatment of incomplete rotator cuff tears. Clin Orthop 1990;254: 64-74.

11. Landis JR, Koch GG. The measurement of observer agreement for categorical data. Biometrics 1977;33: 159-174.

12. Fleiss JL, Cohen J. The equivalence of weighted kappa and the intraclass correlation coefficient as measures of reliability. Educ Psychol Meas 1973;33: 613-619.

13. Rofsky NM, Lee VS, Laub G, et al. Abdominal MR imaging with a volumetric interpolated breath-hold examination. Radiology 1999;212: 876-884.

14. Kataoka M, Ueda H, Koyama $\mathrm{T}$, et al. Contrast-enhanced volumetric interpolated breath-hold examination compared with spin-echo T1-weighted imaging of head and neck tumors. AJR 2005; 184: 313-319.

15. Wetzel SG, Johnson G, Tan AG, et al. Three-dimensional, T1-weighted gradient-echo imaging of the brain with a volumetric interpolated examination. AJNR 2002;6/7: 995-1002.

16. Lauenstein TC, Goehde SC, Herborn CU, et al. Threedimensional volumetric interpolated breath-hold MR imaging for whole-body tumor staging in less than 15 minutes: a feasibility study. AJR 2002;179: 445-449.

17. Mermuys KP, Vanhoenacker PK, Chappel P, Van Hoe L. Threedimensional venography of the brain with a volumetric interpolated sequence. Radiology 2005;234: 901-908.

18. Teefey SA, Hasan SA, Middleton WD, Patel M, Wright RW, Yamaguchi K. Ultrasonography of the rotator cuff. A comparison of ultrasonographic and arthroscopic findings in one hundred consecutive cases. J Bone Joint Surg Am 2000;82: 498-504.

19. Lecouvet FE, Simoni P, Koutaïssoff S, Vande Berg BC, Malgehm J, Dubuc JE. Multidetector spiral CT arthrography of the shoulder. Clinical applications and limits, with MR arthrography and arthroscopic correlations. Eur J Radiol 2008;68: 120-136.

20. Ganguly A, Gold GE, Butts Pauly K, et al. Quantitative evaluation of the relaxivity effects of iodine on GD-DTPA enhanced MR arthrography. J Magn Reson Imaging 2007;25: 1219-1225.

21. Jacobson JA, Lin J, Jamadar DA, Hayes CW. Aids to successful shoulder arthrography performed with a fluoroscopically guided anterior approach. Radiographics 2003;23: 373-378.

22. Matsen FA 3rd. Clinical practice. Rotator-cuff failure. N Engl J Med 2008;358: 2138-2147.

23. Saleem AM, Lee JK, Novak LM. Usefulness of the abduction and external rotation views in shoulder MR arthrography. AJR 2008;191: 1024-1030.

24. Magee T. Can isotropic fast gradient echo imaging be substituted for conventional T1-weighted sequences in shoulder MR arthrography at 3 Tesla? J Magn Reson Imaging 2007;26: 118-122.

25. Lee MJ, Motamedi K, Chow K, Seeger LL. Gradient-recalled echo sequences in direct shoulder MR arthrography for evaluating the labrum. Skeletal Radiol 2008;37: 19-25.

26. Weishaupt D, Wildermuth S, Schmid M, Hilfiker PR, Hodler J, Debatin JF. Virtual MR arthroscopy: new insights into joint morphology. J Magn Reson Imaging 1999;6: 757-760. 